\title{
¿Entes de regulación o control? Imprecisiones del "moderno" esquema de regulación: reflexiones y enseñanzas del caso argentino*
}

\author{
Carolina Nahón ** \\ Nicolás Bonofiglio***
}

SUMARIO: 1. Introducción; 2. La preocupación contemporánea por la regulación; 3. La regulación por la propiedad pública; 4. Una encrucijada: la noción "moderna" de regulación frente a la restatización; 5. La distribución de misiones y funciones en el moderno esquema regulatorio; 6. Diseño institucional de los entes reguladores; 7. ¿Quién controla a los organismos reguladores?; 8. Reflexiones finales.

SUMMARY: 1. Introduction; 2. Current concerns about regulation; 3. Regulation by the public property; 4. A dilemma: the 'modern' concept of regulation $v$. re-statization; 5 . The distribution of missions and functions in the modern regulatory scheme; 6 . Institutional design of the regulatory entities; 7. Who controls the regulatory organisms? 8. Final thoughts.

\footnotetext{
* Artículo recibido en abr. e aceptado en sept. 2007. Los autores agradecen los valiosos comentarios de: Daniel Azpiazu, Karina Forcinito, Cecilia Nahón, Julieta Pesce y Martín Schorr, a quienes se exime de las opiniones, errores y omisiones que pudieran existir.

** Investigadora del Área de Economía y Tecnología de la Facultad Latinoamericana de Ciencias Sociales (Flacso). Maestranda en Sociología Económica (Instituto de Altos Estudios Sociales Universidad Nacional de General San Martín) y licenciada en Ciencia Política (Facultad de Ciencias Sociales - Universidad de Buenos Aires). Becaria del Consejo Nacional de Investigaciones Científicas y Técnicas (Conicet). Domicilio: Ayacucho 551 - Buenos Aires C1026AAC. Argentina. Correo electrónico: cnahon@flacso.org.ar.

*** Investigador del Área de Economía y Tecnología de la Facultad Latinoamericana de Ciencias Sociales (Flacso) - Sede Argentina y profesor de la Universidad Buenos Aires. Maestrando en Ciencia Política (Instituto de Altos Estudios Sociales - Universidad Nacional de General San Martín) y licenciado en Economía (Facultad de Ciencias Económicas Universidad de Buenos Aires). Becario de la Agencia Nacional de Promoción Científica y Tecnológica (Secretaría de Ciencia, Tecnología e Innovación Productiva de la Nación). Domicilio: Ayacucho 551 - Buenos Aires - C1026AAC, Argentina. Correo electrónico: nbonofiglio@flacso.org.ar.
} 
Palabras Clave: regulación; entes reguladores; servicios públicos; Argentina.

KEY WORDS: regulation; regulatory entities; public utilities; Argentina.

Este artículo analiza las particularidades del "moderno" esquema de regulación de los servicios públicos de infraestructura, que se extendió a partir de los procesos de privatización del último cuarto del siglo XX. Diferenciándose de la arquitectura institucional vigente durante el período de prestación estatal (regulación "endógena"), los entes reguladores emergen como el elemento neurálgico de este nuevo esquema. Sin embargo, como se desprende de la experiencia argentina, estos organismos han sido dotados de ambiguas atribuciones regulatorias e inapropiados diseños institucionales, quedando trunca su capacidad real de regulación. A raíz de la difusa delimitación de misiones y funciones e - incluso - frente a las imprecisiones teóricas en la materia, este artículo precisa el alcance de la intervención de los distintos actores del sistema de prestación y regulación: empresas prestadoras, entes reguladores, poderes del Estado (Ejecutivo y Legislativo) y usuarios y consumidores. En este sentido, trabaja sobre los diseños institucionales más apropiados para que los entes desarrollen efectivas capacidades de regulación. El análisis se ejemplifica a partir de la experiencia privatizadora argentina - nacional y subnacional - en los servicios de agua potable y saneamiento y distribución de electricidad.

Regulatory or control agencies? Impreciseness of the 'modern' regulation scheme: thoughts and teachings from the Argentine case This article analyzes the particularities of the public utilities 'modern' regulation scheme, which became predominant during the privatization processes of the last quarter of the twentieth century. In contrast with the institutional framework characteristic of the period of state provision ('endogenous' regulation), the regulatory agencies emerge as the neuralgic element of the new scheme. However, as the Argentine experience demonstrates, these organisms have been endowed with ambiguous regulatory capacities and inappropriate institutional frameworks, and therefore their actual regulatory capacity has been seriously damaged. As a consequence of both this unclear definition of missions and functions and the theoretical imprecision present in this matter, this article attempts to delimitate the extent of involvement of the different actors' scheme: providing companies, regulatory agencies, state powers (Executive and Legislative branches), and both users and consumers. To this respect, the article focuses on the most appropriate institutional frameworks for the development of effective regulatory capacities for the agencies. The analysis is illustrated with examples drawn from the Argentine experience of 
privatization, at the national and sub-national levels, considering the electricity and the drinking water and sewer systems.

\section{Introducción}

La experiencia y la literatura nacional e internacional coinciden en la necesidad de regular la prestación de servicios públicos de infraestructura. ${ }^{1}$ Este fuerte consenso radica particularmente en las características productivas de estos sectores y, en menor medida, en sus efectos en materia de desarrollo económico y bienestar de la población. Sin embargo, los acuerdos iniciales comienzan a reducirse a medida que se intentan precisar los alcances de la noción de regulación. Incluso, en la actualidad, las disidencias suelen evitarse omitiendo la discusión y estableciendo proposiciones genéricas y/o ambiguas respecto de los efectivos ámbitos de intervención de los organismos reguladores de servicios públicos, nacidos a la luz del proceso privatizador. ${ }^{2}$

A más de 15 años de generalizado el proceso de enajenación de activos públicos en América Latina, la discusión reciente se ha centrado en justificar su fracaso en la debilidad de la institucionalidad regulatoria que acompañó a la privatización - entes reguladores deficientes, marcos regulatorios poco específicos o inexistentes; en el incumplimiento de ciertas pautas de procedimiento - omisión del saneamiento previo de las empresas, corrupción en los procesos de venta etc.; y, en definitiva, en la frágil "dotación institucional" de los países en desarrollo que avanzaron en las reformas (Nahón, 2007:3). En este contexto - marcado a su vez por la incipiente ola de restatización en algunos de los países de la región - cobra especial relevancia desentrañar el aspecto regulatorio de la prestación de servicios públicos de infraestructura.

Puesto que el esquema de regulación de este tipo de servicios se define en forma asociada a los arreglos institucionales de prestación vigentes en cada momento histórico, el presente artículo se propone indagar sobre los alcances que ha asumido la intervención regulatoria del Estado en materia de servicios públicos, de forma de contribuir a precisar las particularidades que asume en la actualidad. Para ello, recupera críticamente las principales discusiones teóricas recientes en la disciplina y precisa las misiones, funciones y estructura de

\footnotetext{
${ }^{1}$ A este efecto, consultar: Vickers y Yarrow, 1991; Phillips, 1993; Fiel, 1999; Azpiazu, 2002; Abar, 2003.

${ }^{2}$ En el marco de este artículo se utilizan de modo indistinto los conceptos de "entes reguladores o de regulación", "agencias reguladoras" y "organismos reguladores".
} 
los distintos actores de la regulación pública "moderna"; en particular, aquellas correspondientes a los entes autónomos de regulación y control, en tanto elemento neurálgico de este nuevo esquema. ${ }^{3}$

Si bien el enfoque adoptado es eminentemente teórico, se enmarca en el proceso histórico que le dieron origen, con énfasis en las particularidades del caso argentino. Por medio de pequeños recuadros se incluyen casos prácticos, con la intención de ilustrar, a través de ejemplos regulatorios concretos, la forma en que las prescripciones formales terminan aplicándose en la práctica. La recuperación de experiencias se concentra en los servicios de agua y saneamiento y distribución de energía eléctrica, los que - por su mayor repercusión social y por la inserción institucional de estas reflexiones - fueron relevados en todas las jurisdicciones del nivel subnacional y en la órbita del Estado nacional.

\section{La preocupación contemporánea por la regulación}

Los servicios públicos son aquellas actividades de utilidad pública con características de red cuya garantía de prestación corresponde en forma indelegable al Estado, quien - en tanto poseedor de la titularidad o publicatio - debe velar porque sean prestados en forma regular, uniforme, general y continua (Groisman y Ariño Ortíz, en Lopez y Felder, 1996:10-11). ${ }^{4}$

A los efectos de justificar la necesidad de regular la prestación de los servicios públicos, conviene repasar sus características estructurales:

\footnotetext{
${ }^{3}$ Siguiendo a Abar (2003), la noción "moderna" de regulación se utiliza para delimitar el esquema de regulación vigente, que - asociado al proceso de privatización de los servicios públicos - se distingue de las formas regulatorias que lo precedieron. Si bien no existe un consenso en la literatura sobre la denominación de este esquema (simplemente "nuevo", "regulación por agencias independientes" etc.), sí hay acuerdo en que se trata de una arquitectura institucional regulatoria diferente, cuyo rasgo distintivo son los entes reguladores autónomos. ${ }^{4}$ En tanto "fair de culture et non de nature", la categorización de una actividad como servicio público depende de "las cambiantes necesidades sociales y de los valores dominantes en cada época" (Groisman, 2001:54-55). En la actualidad pueden considerarse como tales, los siguientes servicios: telecomunicaciones; agua potable y desagües cloacales; transporte y distribución de gas natural en redes; producción, transporte y distribución de gas envasado; generación, transmisión y distribución de energía eléctrica; transporte ferroviario (de pasajeros y de carga), por vías fluviales y por caminos; servicios postales, aeroportuarios y transporte aerocomercial.
} 
v economías de escala - a mayor producción es menor el costo unitario, lo que puede volver más conveniente que una única empresa preste el servicio;

v economías de alcance - la prestación de dos o más servicios resulta menos costosa si la realiza una misma empresa;

v elevados requerimientos de inversión fija, con un largo período de maduración;

v demanda relativamente inelástica - las cantidades consumidas son poco sensibles a las variaciones de precios (Cepal, 1995:4-5).

Estas características, especialmente las dos primeras, hacen más conveniente que la prestación de este tipo de servicios quede a cargo de una sola empresa, que constituiría un monopolio "natural". ${ }^{5}$ Desde la perspectiva neoclásica, esto sería suficiente para justificar la existencia de algún tipo de regulación, puesto que se estaría en presencia de "fallas de mercado"; es decir, ante una configuración del mercado alejada de la (construcción analítica de la) "competencia perfecta".

Pese a la justificación neoclásica, la creciente preocupación contemporánea por la regulación de los servicios públicos no se explica, al menos no exclusivamente, por sus características estructurales; vigentes desde que estos sectores comenzaron a ser prestados y que, incluso, constituyen condiciones productivas necesarias para garantizar su funcionamiento eficiente. Por el contrario, responde a un proceso histórico: la privatización de los servicios públicos. Recién en el marco de la ola privatizadora en el último cuarto del siglo XX la función reguladora del Estado es concebida como una práctica en sí misma e involucra la creación de entes reguladores autónomos. Por este motivo, conviene reseñar brevemente las transformaciones de las economías capitalistas que dieron lugar a las privatizaciones y - conjuntamente - a una forma específica de regulación de los servicios públicos de infraestructura.

La superación de la crisis de las economías capitalistas con epicentro en la primera mitad de la década de 1970 implicó una nueva organización de la producción, que - al dejar atrás el modelo fondista - se basó en una mayor segmentación y relocalización de los procesos productivos. Estas transformaciones permitieron la diferenciación de los procesos de trabajo según su complejidad, que condujo a la fragmentación de la clase obrera, al requerir distintos niveles de calificación de los trabajadores. Frente a esta diferenciación, las políticas

\footnotetext{
${ }^{5}$ Cabe aclarar que los avances tecnológicos, que con disímil intensidad se presentan en los distintos sectores, matizan las características estructurales de estos servicios, permitiendo mayores niveles de competencia.
} 
estatales universales perdieron preeminencia, en un contexto de crisis fiscal derivada del estancamiento de las economías y las mayores erogaciones asociadas al aumento del desempleo. De esta manera, el Estado dejó de prestar — o comenzó a hacerlo en forma deficiente - servicios básicos para la población, ya sea salud, educación, seguridad social o servicios de infraestructura. Es decir, de manera gradual aunque no lineal y con distinta intensidad en cada país, se comenzó a desmantelar el Estado de bienestar y, en lo que aquí atañe, a privatizar la prestación de servicios públicos. ${ }^{6}$ Este proceso se dio con mayor intensidad en América Latina, impulsado tanto por los organismos internacionales de crédito - para "resolver" la crisis de la deuda externa y la brecha fiscal — como también por distintas fracciones del capital — interesadas en esos "nuevos mercados".

En el marco de estas transformaciones se consolida la denominada "noción moderna de regulación", que involucra la creación de entes reguladores autónomos enraizados en un esquema regulatorio más amplio (organismos y dependencias estatales). Entonces, no es la mera presencia de monopolios lo que se busca regular por medio de entes autónomos sino, específicamente, la prestación de servicios públicos por empresas privadas. Así, la regulación en sentido "moderno" tiene una especificidad histórica (la privatización a escala), una arquitectura institucional (los entes reguladores) y un régimen legal (marcos regulatorios de orden general y sectorial) que le son propios. En este sentido, se diferencia de - y trasciende a - la visión neoclásica que, al sustentarse en construcciones analíticas abstractas, no logra dar cuenta de las transformaciones reales. De cara a las privatizaciones, para los neoclásicos, la novedad no pasa por la función regulatoria en sí misma — que persiste ligada a recrear un funcionamiento similar al de competencia perfecta - sino por quién la realiza: el ente.

Como se pone de manifiesto, la regulación moderna tiene que enfrentar la tensión entre los intereses privados y el bienestar general, puesto que la búsqueda de lucro por parte de los capitales individuales — en este caso — se realiza en

\footnotetext{
${ }^{6}$ El caso de Estados Unidos requiere una mención especial. Puesto que allí las empresas de prestación de servicios públicos (public utilities) nacen y se consolidan en manos del capital privado, el surgimiento y desarrollo de la estructura regulatoria es de naturaleza diferente al acaecido en países como Gran Bretaña, España, Brasil o Argentina, entre otros. En este sentido, la creación de organismos autónomos para la regulación y el control de la prestación de tales servicios (Independent Regulatory Commissions o, simplemente, Agencies), sucede a una extendida práctica regulatoria por intermedio del Judicial Regulation (primero) y el Direct Legislative Regulation (después) hasta el último cuarto del siglo XIX, en el que a partir de 1870 comienzan a crearse las primeras agencias regulatorias independientes, ligadas exclusivamente a los servicios ferroviarios. Para ampliar sobre esta temática, consultar: Phillips, 1993:127-168.
} 
actividades de alto impacto en las condiciones de vida de la población y el desarrollo económico de los países. En este sentido, es función excluyente - aunque no exclusiva - de los entes reguladores compatibilizar, con miras al beneficio público, la contraposición entre el interés general y el beneficio particular.

En concreto, la regulación no sólo perseguiría aspectos relacionados con criterios de tarifación y de defensa de la competencia - tal como lo sugiere la teoría neoclásica - sino también aquellos vinculados a la cobertura (universalización y tarifas accesibles), la defensa de los derechos de los usuarios y la sustentabilidad del servicio a mediano y largo plazo. En este sentido, sin lesionar los derechos de las empresas prestadoras, la regulación debería trascender la concepción específicamente sectorial y — en su lugar — garantizar que la prestación de los servicios coadyuve al desarrollo económico nacional, a la innovación tecnológica y a la distribución equitativa del ingreso. De esta forma, en la búsqueda de una supuesta neutralidad, la regulación pública no debería limitarse a reducir el riesgo regulatorio y a brindar certidumbre a los inversores (Spiller, 1998:8; Melo, 2002:251), sino que — en su lugar — debería involucrarse en la delimitación de las condiciones de prestación de los servicios, en vistas a alcanzar los mencionados objetivos.

\section{Cuadro 1 \\ Institucionalidad regulatória en Argentina}

Los entes reguladores se difundieron masivamente durante los años 1990. En el nivel nacional, se crearon a inicios de la década para regular los dos sectores objeto de este estudio: agua y saneamiento y distribución de energía eléctrica. En el nivel subnacional, el proceso alcanzó a 18 de las 24 jurisdicciones, que, hacia mediados o fines del decenio, establecieron entes sectoriales o multisectoriales. En todos los casos, la creación de estos organismos estuvo estrechamente vinculada a los procesos de privatización. De esta forma, los pocos casos en los que coexisten entes y empresas de prestación estatal se explican por la restatización de los servicios (Entre Ríos — electricidad — y Buenos Aires, Santa Fe, Tucumán y Estado nacional — agua y saneamiento) o bien por procesos de privatización "fallidos", en los que los entes se habían creado en forma previa a la efectiva transferencia de los servicios (Córdoba - sector eléctrico y Jujuy — agua y saneamiento).

En el nivel subnacional, algunas jurisdicciones no crearon organismos autónomos de regulación y control, de modo que estas funciones se mantuvieron en los ministerios o secretarías desde donde se venían desarrollando históricamente. En estos casos, se comprueba que las leyes (o proyectos) que contemplaban la instrumentación de este tipo de agencias estaban asociadas a la privatización del servicio. Una vez más, al fracasar esta última, se dejó de lado la institucionalización de los respectivos organismos (Santa Fe, Córdoba y Misiones — energía eléctrica y Río Negro — agua y saneamiento, aunque en este último caso existe una dirección provincial responsable de la regulación y el control). 


\section{La regulación por la propiedad pública}

Si la nueva noción de regulación nace con la privatización, emerge la pregunta sobre cómo era el régimen anterior, es decir, aquél que regía cuando los servicios públicos eran prestados por empresas estatales. ¿Es correcto afirmar que las empresas públicas convivían con la "desregulación", esto es, que su prestación no era regulada por el Estado?

La respuesta es compleja. Sin embargo, un camino para su resolución es distinguir entre dos nociones de regulación de distinto alcance y asociadas a dos momentos históricos diferentes. Si por "regulación" se entiende la capacidad estatal de definir reglas de cumplimiento obligatorio en el marco de una política de Estado previamente determinada en las esferas del Ejecutivo, es innegable que la operación estatal de los servicios públicos se halla regulada desde sus inicios. En América Latina, así lo certifica la práctica de las empresas estatales durante la fase de "industrialización por sustitución de importaciones", período en el cual la expansión de los servicios se enmarcaba en una estrategia de desarrollo regional y en la mejora de las condiciones de vida de la población. Desde esta perspectiva, por estar los servicios a cargo del propio Estado, la regulación no era otra cosa que "regulación endógena" o bien "regulación por la propiedad pública". ${ }^{7}$ Es decir, ya sea que actuase por intermedio de la propia empresa (Ilpes, 1999:144) o bien que dependiera de los departamentos u organismos de la burocracia ejecutiva (Melo, 2002:249) se concretaba alguna forma de "autorregulación" de la prestación pública de servicios esenciales. ${ }^{8}$

Desde una concepción opuesta, que se enmarca en el desempeño crítico de las empresas estatales de la región durante la década del 1980 (en particular hacia el final del decenio), se sostiene que la regulación por la propiedad pública presenta serias deficiencias. La profunda crisis de las economías de

\footnotetext{
${ }^{7}$ En palabras de Melo (2002:249, traducción y bastardilla propias): "Las Agencias Reguladores independientes representan una nueva forma de regulación pública de sectores económicos, substituyendo el ejercicio de actividades de regulación directamente por departamentos $\mathrm{u}$ órganos de la burocracia ejecutiva. En este tipo de regulación endógena o implícita, los órganos estaban bajo el mando directo de los gobiernos, que también detentaban la propiedad de empresas monopolistas. La regulación por la propiedad pública fue durante décadas el principal medio de regulación en el área de infraestructura...".

${ }^{8}$ Por el contrario, Abar (2003:11) no considera los mecanismos de intervención directa como forma de regulación. De este modo, sostiene: "Si la actividad regulatoria estatal constituye una especie de intervención (indirecta) en el orden económico, la asunción de la explotación de actividad económica por el Estado o por su ente controlado no caracteriza intervención reguladora".
} 
América Latina, producto - entre otros - del abultado endeudamiento externo, condujo a que las empresas estatales registraran enormes déficits de inversión ${ }^{9} \mathrm{y}$, progresivamente, fuesen utilizadas como herramientas estabilizadoras (política antiinflacionaria), fiscales y de empleo; y como vehículos para la transferencia de recursos públicos (por medio de subsidios directos e indirectos) al capital concentrado local. A partir de aquí, los autores de esta corriente sostienen que el Estado es incapaz de cumplir adecuadamente la función regulatoria. Como correlato, se limitan a justificar la transferencia de las obligaciones del Estado en materia de prestación como respuesta a esta "ineficiencia" estatal (Massey en Orlansky, 1998:835-836) o bien debido a la dificultad del Estado de realizar conjuntamente las funciones de regulación, prestación y control (Fiel, 1999; Abar, 2003:11).

\title{
Cuadro 2
}

\section{La expansión de la red de gas y el fomento al capital privado en Argentina}

\begin{abstract}
En el marco del modelo de industrialización por sustitución de importaciones, la regulación por la propiedad pública de los servicios públicos incluyó objetivos que excedían el marco específicamente sectorial.

En relación con el desarrollo de la cobertura, la política de ampliación de la red de distribución de gas natural resulta ilustrativa. La empresa estatal YPF, a partir de la creación de Gas del Estado (1946), dejó de ventear el gas y pasó a transferírselo a precios ínfimos, lo que permitió montar una amplia red de distribución de gas natural sin que esto implicara elevados costos de ingreso al servicio para los nuevos usuarios. Como resultado, entre 1960 y 1991, el consumo de gas natural se incrementó a una tasa media anual de 12,2\% y, a principios de Ios años 1990, el 45\% de los hogares accedía al gas natural en su domicilio (Azpiazu y Schorr, 2001:6). De esta manera, el desarrollo de una de las redes de gas más grandes de América Latina fue producto de una política estatal explícita de tarifas "subsidiadas".

En el marco de la política de promoción industrial, la Ley 14.781, impulsada por el gobierno de Arturo Frondizi (1958-62), contemplaba el suministro preferencial de electricidad para las empresas radicadas en el país. Concretamente, otorgaba un subsidio tarifario con el objeto de fomentar la promoción de actividades manufactureras, específicamente, industrias "pesadas" y/o electrointensivas.
\end{abstract}

\footnotetext{
${ }^{9}$ En el caso argentino, hay que destacar que si bien en la década de 1980, en el marco de la crisis de la deuda, se produjo una caída de la inversión pública, la privada se realizó mayoritariamente con recursos públicos otorgados por los distintos regímenes promocionales (Basualdo, 2006:253-255).
} 
De todas formas, con independencia del momento histórico desde el que se analice este fenómeno, la regulación por la propiedad pública estaba imbricada en la definición de la política estatal en sentido amplio. En este sentido, en función del régimen de acumulación vigente, se incluían objetivos que excedían el marco específicamente sectorial (en favor - o no - del desarrollo sustentable de los servicios, según el momento histórico).

Ahora bien, si por "regulación" se entiende lo que aquí se ha denominado "noción moderna de regulación", durante la etapa de prestación estatal es claro que no existía. En este contexto, ¿cómo se justifica el cambio en la noción de regulación? ¿Esto es, el desplazamiento del locus institucional en que ella se desenvuelve? ¿A qué se debe este pasaje de atribuciones entre empresas del Estado, ministerios o secretarías del Poder Ejecutivo y agencias autónomas de la administración pública central? Más allá de las controversias sobre el pasado, es claro que el cambio de modelo regulatorio se origina con la emergencia de prestadores privados. De esta forma, si bien es posible afirmar que siempre existió alguna forma de intervención estatal regulatoria, lo novedoso es el lugar institucional en el que ella se desenvuelve, su orientación sectorial en vez de sistémica y — por supuesto - los actores regulados.

\section{Una encrucijada: la noción "moderna" de regulación frente a la restatización}

La pregunta sobre la necesidad, utilidad u oportunidad de la regulación también puede formularse respecto del futuro inmediato. Es decir, para el caso de los servicios públicos que vuelven a manos del Estado ¿la regulación pública en su acepción moderna seguiría justificándose? Sobre este asunto existe un fuerte consenso: la presencia de entes reguladores autónomos no debe desaparecer (Stark, 2001:154; Abar, 2003:11-18; ACIJ, 2007; UUC, 2007).

A partir de este acuerdo es posible pensar que el esquema regulatorio moderno trasciende el régimen de propiedad. La experiencia ha demostrado que la regulación por la propiedad pública no siempre ha jerarquizado el desarrollo de los servicios públicos, específicamente, tras el fin de la industrialización sustitutiva. Asimismo, desde la expansión de la prestación privada de los servicios tuvieron lugar "fallas regulatorias", tanto o más difíciles de revertir que las que se derivaban de la regulación endógena. ${ }^{10}$ De esta for-

\footnotetext{
${ }^{10}$ Las denominadas "fallas regulatorias" son — básicamente — de dos tipos: económicas y políticas. Entre las primeras se encuentran aquellas derivadas de la información asimétrica -
} 
ma, en el marco de la incipiente restatización de servicios, la regulación por medio de este tipo de entes, si bien no garantiza la reversión de esta tendencia es - al menos - auspiciosa, en tanto revaloriza la función de organismos autónomos como herramienta para evitar la intromisión de criterios regulatorios ajenos al funcionamiento adecuado de los sectores.

El conocimiento técnico sobre el sector regulado y la independencia (tanto del poder político como del prestador) que debería caracterizar a estos organismos, les permite afrontar la función regulatoria desde un lugar estratégico. Sin embargo, no por ello dejan de verse afectados por los "problemas de agencia", "asimetrías de información" y "cooptación bifronte" propios de la interacción con múltiples actores: sociedad, usuarios, empresas y dependencias del Poder Ejecutivo. En este sentido, la regulación en su acepción moderna no es más que la arquitectura institucional que - en el marco de las democracias actuales - está mejor preparada para enfrentar airosamente el desafío de la regulación y el control de la prestación — pública o privada — de servicios esenciales. ${ }^{11}$

En particular, en el caso de las empresas públicas, para que la tríada de actores de naturaleza estatal - empresas prestadoras, Poder Ejecutivo y ente regulador - no contribuya a la burocratización del quehacer regulatorio por medio de una recurrente superposición de actuaciones, es preciso no sólo que se definan en forma específica las misiones y funciones de las partes, sino que - paralelamente - se prevean instancias de coordinación y articulación que contemplen algún grado de participación ciudadana. La escisión de responsabilidades es inherente a los "pesos y contrapesos" del Estado de derecho - Poder Legislativo, Ejecutivo y Judicial - y la especificidad de la regulación de los servicios públicos de infraestructura la hace altamente recomendable.

entre regulador y regulado, costos administrativos de la regulación y posible inequidad o ineficiencia de los resultados derivadas de su intervención (Gómez, 2006:5). Dentro de las segundas, entran en juego los "problemas de agencia", resultantes de la cadena de principales y agentes (ciudadanía, Poder Ejecutivo, ente regulador, empresa regulada y usuarios y consumidores) involucrados en todo esquema de prestación, regulación y control. Para ampliar sobre las derivaciones de la teoría del principal-agente sobre la actuación de los entes reguladores, ver: Ross, 1973; Porto y Urbiztondo, 1993.

${ }^{11}$ En el caso de la prestación pública, la elección de la forma jurídica (empresas del Estado o sociedades anónimas) no invalida la opción por el esquema moderno de regulación, aunque sí sea relevante en términos del funcionamiento interno de las empresas (regímenes de compras, contrataciones de personal etc.) y control externo por medio de otros organismos del Estado (auditorías, sindicaturas, tribunales de cuentas etc.). 


\section{Cuadro 3}

\section{La restatización de servicios y los entes de regulación en Argentina}

Al margen del caso de Tucumán en el servicio de agua y saneamiento (1997), las seis restatizaciones que tuvieron lugar en los sectores objeto de este artículo se sucedieron a partir del año 2002, en el nuevo contexto macroeconómico originado por el colapso del régimen de convertibilidad (1991-2002). Las jurisdicciones del nivel subnacional que avanzaron en este sentido (Buenos Aires — en ambos sectores, Santa Fe y Tucumán agua y saneamiento y Entre Ríos — distribución de electricidad) no involucraron una modificación del esquema regulatorio hasta entonces vigente: Ios respectivos entes reguladores se mantuvieron en pie, conservando sus misiones y funciones e, inclusive, su capacidad sancionatoria.

Con un matiz diferente, pero no poco significativo, en el nivel nacional se restatizó el servicio de agua y saneamiento anteriormente a cargo Aguas Argentinas S.A. En la actualidad, el sistema sanitario más grande del país, con un área de concesión de más de nueve millones de habitantes, se encuentra en manos de una sociedad anónima mayoritariamente estatal. A diferencia de lo acaecido en el nivel subnacional, el ente regulador del sector (Etoss) fue formalmente reemplazado por uno nuevo (Eras), cuyas atribuciones quedaron limitadas al control del servicio. Sin embargo, cumplido el año de gestión de la empresa estatal, el Eras aún no ha sido constituido, de forma que ésta opera sin ningún tipo de control sectorial externo.

De esta forma, si bien los entes nacieron en forma asociada al proceso privatizador, este esquema institucional se ha mantenido a pesar de las restatizaciones, de modo que, en este nuevo contexto histórico, vuelve a dejarse atrás el modelo de regulación por la propiedad pública.

\section{La distribución de misiones y funciones en el moderno esquema regulatorio}

La delimitación de las atribuciones de los entes de regulación es la aspiración central de este artículo, en tanto se trata del elemento neurálgico del esquema moderno de regulación. Puesto que la literatura es escasa y breve la experiencia práctica en la región, a continuación se precisan y delimitan las misiones y funciones de estos organismos, anteponiéndolas a aquellas propias de los restantes actores: poderes del Estado y empresas prestadoras.

\section{Los poderes del Estado: Ejecutivo y Legislativo}

Como ya fue mencionado, la titularidad de los servicios públicos le corresponde al Estado - nacional o subnacional - , quien por este motivo es aquél que debe garantizar una prestación regular, uniforme, general y continua. Esto no implica que deba asumir por sí mismo la prestación del servicio, sino que, en determinadas circunstancias históricas, puede optar porque una empresa pri- 
vada quede a cargo de la prestación. En este caso, el Estado "concede" el servicio; es decir, ejerce, en su carácter de titular, su poder concedente.

De esta forma, debe quedar claro el papel indelegable del Estado en la definición de la política sectorial de mediano y largo plazo, por intermedio de los ministerios o secretarías de referencia. Esto es, con independencia del modelo regulatorio que se adopte y más allá de que la prestación recaiga en empresas públicas o privadas, la planificación - es decir, la definición de las metas y objetivos de desarrollo del servicio - es una atribución exclusiva del Poder Ejecutivo. A este efecto, debería seguir las pautas inscriptas en un régimen de servicios públicos de orden general y en los respectivos marcos regulatorios sectoriales, previamente sancionados por ley del Congreso, nacional o subnacionales. ${ }^{12}$ En este sentido, en los marcos regulatorios debieran definirse aspectos clave, tales como los deberes del Estado, la finalidad de la prestación, los tipos de propiedad admitidos y la institucionalidad regulatoria, que, según las particularidades de contexto y las orientaciones propias de cada gestión gubernamental, el Ejecutivo de turno plasmará en la definición de las estrategias y políticas de infraestructura.

\section{Las empresas prestadoras}

Conviene precisar el papel de las empresas prestadoras. Sean éstas públicas o privadas, deben ajustarse a los lineamientos de política definidos en el marco del Poder Ejecutivo. Para ello, es recomendable la firma de contratos de concesión (empresas privadas) o licencias o permisos (empresas públicas). ${ }^{13} \mathrm{Si}$ bien la delimitación de derechos y obligaciones de las partes es una práctica común en la prestación privada de servicios públicos, son muy pocos los casos en que, frente a la operación estatal, se corrobora la institucionalización de compromisos de prestación; lo que - naturalmente - impide una correcta delimitación de funciones y atribuciones entre los distintos actores del sistema. En cualquier caso, en los contratos deberían quedar plasmados los lineamientos para el sector, tanto en términos de la sustentabilidad del servicio

\footnotetext{
${ }^{12}$ Es deseable que la sanción de la normativa regulatoria se instrumente por medio de leyes en lugar de decretos. Si bien la intervención parlamentaria no necesariamente garantiza un adecuado estudio de la problemática objeto de regulación, la participación del Poder Legislativo propende al debate público y hace descansar a la normativa sobre una voluntad colegiada.

${ }^{13}$ En adelante, salvo aclaración contraria, toda mención a compromisos "contractuales" aludirá a los "contratos" o las "licencias y permisos", según que se esté refiriendo a prestadores privados o públicos, respectivamente.
} 
- que en algunos sectores comprende el uso de recursos no renovables como de la viabilidad financiera de los planes de expansión, criterios de tarifación y metas de calidad. Puesto que la delegación de la prestación tanto a empresas públicas como privadas - por lo general se extiende por varios años, los compromisos plasmados en los contratos, necesariamente, deberán volverse más específicos por medio de sucesivos planes de mejora, en los que se puntualizarán — para períodos más reducidos de tiempo — las grandes metas pactadas inicialmente. Es justamente aquí en donde entran en escena los entes reguladores.

\section{Los entes reguladores}

Según se alude en la literatura en la materia - y se corrobora en las normativas vigentes de varios países - los entes reguladores independientes de la administración pública central, responsables de intervenir en materia de servicios públicos, asumen - en términos generales - las siguientes cuatro funciones básicas: regulación, control, fiscalización y sanción. ${ }^{14}$ En este sentido, la definición de los alcances de las tres últimas funciones no presenta mayores inconvenientes.

En su carácter de organismos de control, los entes reguladores deberían perseguir el cumplimiento por parte de los prestadores de la normativa (marcos regulatorios) y de los compromisos asumidos en los contratos originales y en los planes de mejora para subperíodos contractuales específicos. En este sentido, el ente debe implementar un sistema de contabilidad regulatoria, ${ }^{15}$ demandar informes de gestión, balances y cuadros tarifarios; y certificar el cumplimiento de los planes de obra y las normas de calidad. Es decir, realizar todas aquellas tareas necesarias para corroborar el efectivo cumplimiento de los prestadores de los compromisos legales y contractuales. Por consiguiente, en los casos de prestación estatal, donde — por lo general — no se formalizan

\footnotetext{
${ }^{14}$ A este efecto, consultar: Herrero, 2002; Abar, 2003, Melo, 2002.

${ }^{15}$ La contabilidad regulatoria es la metodología por medio de la cual los entes reguladores disponen criterios uniformes para que las empresas reguladas imputen costos e ingresos (incluyendo, por supuesto, inversión), de forma de evitar la doble internalización de costos en tarifas. En definitiva, la intención es capturar en forma separada los gastos provenientes de servicios regulados básicos, de las prestaciones asociadas y de los servicios no regulados cuando correspondiere - con el fin de encausar las tareas de control propias de estos organismos y - de esta manera - disminuir las asimetrías de información asociadas al esquema regulatorio moderno.
} 
los derechos y obligaciones de las partes, la función de control del ente queda desdibujada y pasa a depender sobre todo - tal como se verá a continuación - de sus capacidades de regulación.

La fiscalización es uno de los medios para poner en práctica la función de control. De esta manera, el ente deber autorizar la inspección in situ de las obras, plantas y oficinas de las prestadoras, así como habilitar la práctica del "examen individualizado" (screening) de productos, procesos y productores a los efectos de medir de manera sistemática y uniforme el cumplimiento de los requisitos contractuales o evaluar en forma simultánea varios factores (Majone y La Spina, 1993:222).

La sanción es la capacidad por medio de la cual los entes reguladores imponen penas sobre los incumplimientos contractuales o normativos de los prestadores. Ya sea que los entes sean los responsables de definir el reglamento de sanciones o bien cuando éste es pautado en instancias de estatus jerárquico superior, es la capacidad sancionatoria aquella que le otorga sentido a las dos funciones previas: control y fiscalización.

Frente a prestación privada, es clave que el monto de la sanción sea lo suficientemente significativo para evitar que a la empresa le sea más rentable el incumplimiento. Distinta es la situación cuando la prestación recae en empresas de capital estatal. Si bien resulta prudente que el Estado no se sancione a sí mismo - debido a que implicaría una doble imposición para la sociedad - el sistema de control y fiscalización no debería dejar de descansar en alguna forma de estructura sancionatoria (Nahón, 2006:19). ${ }^{16}$ Para ello, debería considerarse que las multas y sanciones por incumplimientos afecten en forma directa a los miembros del directorio de las empresas estatales, incluyendo sus patrimonios. En cualquier caso, los montos recaudados por la vía sancionatoria deberían tener - tal como ocurre en la actualidad - los siguientes destinos: los usuarios directamente afectados por el incumplimiento, el ente regulador o rentas generales, según si la multa fue ocasionada por problemas de facturación, calidad y prestación individualizables, en el primer caso; o por retrasos en los planes de obra, constitución de garantía u otros incumplimientos de la normativa o el contrato de alcance general, en los dos últimos casos.

Respecto de las funciones de control, sanción y fiscalización existe un extendido consenso - en la literatura académica y la normativa - sobre que deben recaer en forma exclusiva en los entes reguladores. El problema surge

\footnotetext{
${ }^{16}$ En Argentina, por ejemplo, dos dictámenes de la Procuración del Tesoro de la Nación (441/ 04 y 053/05) — sustentándose en el "principio de cooperación y unidad de acción" — inhiben la aplicación de multas y sanciones por parte del Estado sobre entes "enteramente estatales".
} 
cuando se pretende dilucidar los alcances de las atribuciones de regulación de estos entes. ¿Cuándo un ente regulador es — paradójicamente — regulador? ¿La regulación se limita a la mera reglamentación o, por el contrario, la incluye como una de sus atribuciones? En este sentido, cobra relevancia la posibilidad de precisar los alcances de ambas nociones: regulación y reglamentación.

Las tareas de reglamentación son propias de toda institución con atribuciones específicas sobre un sector e involucran funciones ejecutivas, es decir: administrativas y de gestión. En este sentido, por medio de resoluciones, se orientan a "disciplinar una actividad mediante la emisión de comandos normativos, de carácter general, aún cuando tengan un alcance meramente sectorial" (Abar, 2003:15). Es decir, a operacionalizar criterios generales - en términos de calidad, planes de mejoras y obras, entre otros — definidos en normas de estatus jurídico superior sobre la base del conocimiento técnico del sector propio del ente regulador. ${ }^{17}$

Por su parte, las tareas de regulación propiamente dichas, si bien incluyen aquellas de reglamentación, constituyen la capacidad del organismo de intervenir en cuestiones centrales del proceso de prestación. En este sentido, por medio de sus atribuciones regulatorias, los entes deberían poder dictar los reglamentos de audiencias públicas y sanciones; confeccionar los pliegos, contratos, licencias y permisos; ejercer algún tipo de control antimonopólico (en función de las particularidades sectoriales y en forma articulada con el órgano responsable de defensa de la competencia); calcular y definir el valor de las tarifas y establecer el plan de inversiones. ${ }^{18}$ En los casos de prestación privada, estos organismos, a su vez, deberían seleccionar al consorcio operador (tras una licitación pública), intervenir activamente en los procesos de renegociación contractual y, en último término, hasta contar con la capacidad de rescindir los contratos frente a incumplimientos explícitamente pautados (facultad transferida por el poder concedente).

\footnotetext{
${ }^{17}$ Por ejemplo, la delimitación precisa, en función de prescripciones normativas genéricas inscriptas en los marcos regulatorios, de los niveles de arsénico permitidos para que el agua sea considerada potable o los márgenes de tensión autorizados en el caso de la energía eléctrica. ${ }_{18}$ Por ejemplo, el organismo regulador de agua y saneamiento en Inglaterra y Gales, Water Services Regulation Authority (OFWAT) tiene entre sus atribuciones la fijación de las tarifas máximas a las compañías por un período de cinco años. Por su parte, la Comisión de la Industria del Agua de Escocia, en donde la prestación recae en una empresa de propiedad estatal, debe regular precio, calidad, estándar del servicio y calidad de activos. Para ampliar, ver: $<$ www.ofwat.gov.uk $>$ y $<$ www.watercommissioner.co.uk $>$.
} 


\section{Cuadro 4}

\section{Prácticas regulatorias en Argentina}

En los niveles nacional y subnacional los entes reguladores encuentran restringido su campo de intervención regulatoria, tanto por limitaciones de tipo estructural como por imprecisiones normativas. Las limitaciones estructurales comprenden cuestiones presupuestarias, de personal y edilicias, que - en definitiva - son un termómetro de la voluntad política en esta materia. Así, se corroboran esquemas de financiamiento híbridos y/ o desvinculados de las prescripciones normativas, entes sin estructuras orgánicas definidas ni suficiente personal, entre otras trabas operativas.

Las imprecisiones normativas afectan el campo de intervención de estos organismos. Muchas veces, los marcos normativos no cuentan con decretos reglamentarios y, en los pocos casos en que estos existen, no siempre delimitan con precisión qué debe entenderse por "intervenir", "dictaminar", "aprobar", "establecer", "evaluar" o "elaborar ad referéndum del Poder Ejecutivo". El problema radica en que — según la interpretación que se adopte en cada caso - estos conceptos pueden significar una atribución exclusiva del ente sobre una determinada materia, una atribución compartida con el ministerio o secretaría de referencia (es decir, con la autoridad de aplicación) 0, incluso, una mera intervención administrativa en lugar de una capacidad regulatoria. De este modo, el alcance de sus atribuciones (misiones y funciones) se vuelve difícil de precisar: iserá que por "aprobar" "establecer" o "definir" deberá entenderse, simplemente, "poner el sello habilitante" sobre lo que define la autoridad de aplicación? En otras palabras, ¿hasta qué punto son reguladores los entes reguladores?

En la práctica, los entes casi no han tenido intervención en las definiciones tarifarias o en los procesos de renegociación. Ya sea porque las propias leyes marco no les han asignado estas funciones (Catamarca y Corrientes en agua y saneamiento) o bien porque les han sido arrebatadas por la autoridad de aplicación (Córdoba y Estado nacional en agua y saneamiento) su papel se ha reducido, en el mejor de los casos, al de simples asesores externos, con voz pero sin voto. En definitiva, en el marco de las funciones ejecutivas inherentes a la existencia de cualquier dependencia administrativa, los entes se han limitado a definir cuestiones reglamentarias básicas (como la calidad del servicio), perdiendo las atribuciones regulatorias que le son propias.

A los efectos de zanjar toda disyuntiva sobre la delimitación de funciones entre los entes reguladores y las autoridades de aplicación, las provincias de Buenos Aires — en ambos sectores — y de Santiago del Estero en agua y saneamiento — han dado un osado paso: concentraron formalmente las atribuciones de regulación en las autoridades de aplicación, legando en los entes las funciones de control, fiscalización y sanción. Si bien ésta no resulta una práctica deseable en términos de las aspiraciones de la noción moderna de regulación, es - al menos - un paso firme en el sentido de clarificar los ámbitos de intervención de los distintos actores: Poder Ejecutivo, entes reguladores y empresas prestadoras.

En términos regulatorios, el límite a sus atribuciones está dado por las prescripciones de los marcos regulatorios y, naturalmente, por la política sectorial que se defina en el marco del Poder Ejecutivo. De esta forma, debe quedar claro que las atribuciones regulatorias de los entes terminan allí donde comienzan - y deben guiarse por - las políticas de Estado. Resulta funda- 
mental, entonces, que la normativa aborde de la forma más precisa posible la delimitación de funciones entre los entes - en tanto que organismos autónomos de regulación y control - y los ministerios o secretarías de referencia del Poder Ejecutivo - como autoridades de aplicación y, por tanto, responsables de la definición, en nombre del Ejecutivo, de la política sectorial.

La nueva noción de regulación involucra un trade off entre la autonomía de las agencias - en tanto que garante de la "credibilidad regulatoria" — y el "déficit de legitimidad" que se deriva de la delegación de poder regulatorio en manos de actores no electos (Melo, 2000:10). Respecto de la autonomía, se trata de la variable fundamental para garantizar la especificidad técnica y sectorial en el marco de esta nueva noción de regulación. Es, en definitiva, el elemento distintivo del nuevo modelo y aquello que permite pensar en una intervención regulatoria - que si bien no deja de estar sometida a lineamientos políticos - tiene capacidad de decidir aspectos de largo plazo en función de las particularidades sectoriales de cada servicio y - de esta forma - conducir el proceso de control, fiscalización y sanción según surja de la normativa vigente. En relación con el denominado déficit de legitimidad de estas agencias, Majone (1999) señala: "este déficit puede ser minimizado si una adecuada estructura de gobernanza fuera creada y permitiese una mayor responsabilidad sobre sus decisiones (por medio de la revisión judicial, requisitos procedimentales, supervisión por comité, participación pública...)" (en Melo, 2000:31, traducción propia).

\section{Diseño institucional de los entes reguladores}

Una vez delimitadas las misiones y funciones de los distintos actores del esquema moderno de regulación y - en particular - de los entes, se precisan algunos aspectos clave en términos de gobernanza y accountability (rendición de cuentas) de estas agencias independientes que, si bien no representan una solución mágica a los "problema de agencia" y "fallas regulatorias", pueden contribuir a mejorar su desempeño.

Con independencia del tipo de prestador (público o privado) la literatura recomienda apuntar a diseños institucionales capaces de ponerle coto a la denominada "cooptación bifronte" (Vispo, 1999). Esta captura en sentido doble implica, por un lado, la cooptación estándar que se dirige desde las empresas reguladas y los grupos de interés hacia la agencia reguladora. Y, por el otro, el “oportunismo gubernamental" (Spiller, 1998:5-9) o “expropiación regulatoria” 
(Urbiztondo, Artana y Navajas, 1998:8), que significa el quiebre de la independencia de la figura regulatoria desde el propio Estado - sea por intermedio directo del Poder Ejecutivo como de alguno de sus ministerios o secretarías o, peor aún, por la influencia misma que puede ejercer el poder político. $^{19}$

Para limitar los alcances de la cooptación existe un fuerte consenso respecto de que los entes deben gozar de efectiva autarquía financiera y autonomía funcional. En el primer sentido, se recomienda que elaboren sus respectivos presupuestos, que - salvo excepción fundada - deberán ser de carácter intangible. En términos del origen del financiamiento, la recomendación varía en función de la estructura del mercado a regular. Cuando más de una empresa esté bajo su tutela — sean varias firmas de una misma cadena productiva o dos o más firmas monopólicas (de un mismo sector o no) —, se recomienda el financiamiento por medio de tasas de fiscalización y control pagadas por las empresas o sus usuarios a través de las tarifas. De esta manera, como los fondos del ente no van a estar directamente ligados a la facturación/ tarifa de una única empresa ni tampoco a los recursos públicos, las posibilidades de cooptación se reducen. Distinto es el caso de un ente que regula una única empresa: acá cualquier arreglo parece incentivar la cooptación en beneficio de aquél que transfiere los recursos. Para enfrentar esta dificultad podría utilizarse un sistema de financiamiento mixto, en el que los fondos provengan tanto desde la empresa como desde el Tesoro.

Así, cuanto menor sea el número de empresas reguladas, la captura del ente resultará más difícil de evitar: quien gire los recursos al ente - Tesoro y/ o empresas reguladas - tendrá mayores posibilidades de cooptar al organismo, en un contexto en el que la atomización de los aportantes resulta muy difícil. Asimismo, con independencia de la modalidad que se elija para el financiamiento de estos organismos, el costo de la regulación, en última instancia, es afrontado en su totalidad (varias empresas) o en parte (única empresa) por los usuarios y consumidores. En este último caso, dado que parte de los recursos provienen de rentas generales, el resto de la población es cofinanciadora de la prestación del servicio, sea o no beneficiaria.

Respecto de la autonomía funcional, un elemento pocas veces recuperado por la literatura doméstica - y previamente comentado en este artículo -

\footnotetext{
${ }^{19}$ Un riguroso análisis de la forma en que se ha desplegado este tipo de cooptación en el marco del programa de privatizaciones de servicios públicos dependiente del Estado nacional, puede encontrarse en Azpiazu, 2001.
} 
es el denominado "recurso jerárquico o de alzada". Puesto que en América Latina - por lo general - los entes reguladores son entidades autárquicas con personería jurídica propia, pero que funcionan en el "ámbito" del Poder Ejecutivo, sus resoluciones pueden ser recurridas por vía administrativa ante el directorio del organismo, el ministerio o secretaría de referencia y, en último término, ante el Poder Ejecutivo. Si bien la vía judicial - naturalmente - se encuentra habilitada, al no ser ésta la única vía recursiva posible, da lugar a que las empresas abusen del recurso jerárquico que, a diferencia de la vía judicial, no posee costo alguno. En este contexto, la autonomía funcional del organismo se encuentra plenamente vulnerada. Sus decisiones regulatorias y sancionatorias pueden ser permanentemente cuestionadas por órganos administrativos de superior jerarquía pero menor conocimiento especializado, quienes con frecuencia priorizan el corto plazo y los intereses extrasectoriales. Si bien esta práctica resulta razonable a los efectos de proteger los derechos de usuarios y consumidores e, incluso, de los particulares afectados, no parecería prudente que las empresas controladas gozasen de semejantes beneficios - $\mathrm{o}$, al menos, que esto sea así para la totalidad de las resoluciones de los entes. En este sentido, limitar las causales habilitadas para la presentación de recursos y hacer descansar el grueso de las apelaciones en el sistema judicial parecería ser una fórmula viable para respetar las garantías de los inversores y la independencia regulatoria. ${ }^{20}$

Asimismo, a los efectos de garantizar la autonomía y la capacidad técnica de los entes reguladores, sus autoridades deberían ser elegidas conforme a criterios de idoneidad e independencia (respecto de los consorcios operadores y del poder político) por medio de concursos públicos de oposición y antecedentes, que contemplen las calificaciones de los aspirantes y permitan la participación ciudadana. De igual forma, su personal debería estar enmarcado en una carrera administrativa pública, que garantice la prevalencia de criterios técnicos y pautas de procedimientos acordes con la envergadura de la tarea a su cargo.

En particular, respecto a su independencia del poder político, estos organismos no deberían poder ser intervenidos por el Poder Ejecutivo, salvo en casos excepcionales y frente a causales previa y explícitamente determinadas. A su vez, sería aconsejable que sus directores gozaran de intangibilidad en las

\footnotetext{
${ }^{20}$ El sistema regulatorio vigente en Estados Unidos descansa - como aquí se sugiere - en el Poder Judicial. De esta forma, tanto en el nivel federal como en los estaduales, no está contemplada la alzada administrativa sobre las decisiones de los entes reguladores (Herrero, 2002:142).
} 
remuneraciones y que actuasen como funcionarios públicos con dedicación exclusiva durante un período de no más de cuatro años, renovable por única vez. Con la intención de resguardar su autonomía frente a las prestadoras, debería regularse un período de caución. En este lapso, los ex directores no estarían autorizados a asumir cargos de responsabilidad en las empresas reguladas ni deberían estar vinculados a consultoras que les brindaran servicios de asesoría. Incluso, para aquellos aspirantes a directores que hubieran tenido vinculación previa con las empresas prestadoras, debería regularse un período de inhabilitación en el que no estarían autorizados a competir por el cargo.

Mientras estén en funciones, los directores deben tomar sus decisiones de forma colegiada y seguir - en todos los casos - los requisitos procedimentales definidos, garantizando particularmente la transparencia de los procesos y las decisiones adoptadas. Asimismo, es imprescindible que estos organismos contemplen la participación de las asociaciones de usuarios y consumidores. ${ }^{21}$

La autarquía financiera y la autonomía funcional debieran regir tanto en

\section{Cuadro 5}

\section{Los directorios de los organismos en Argentina}

La composición de los directorios es un aspecto clave de la puesta en marcha del sistema de regulación y control. Las recurrentes modificaciones normativas en esta materia y — peor aún — los reiterados incumplimientos de las prescripciones finalmente adoptadas, dan cuenta de uno de los aspectos más endebles de la aplicación práctica del esquema moderno de regulación.

En esta materia, se corroboran irregularidades de todo tipo. En la mayoría de las jurisdicciones, Ios directorios han permanecido incompletos, quedando varias veces vacantes más de uno de sus cargos (Estado nacional — distribución de energía eléctrica; Córdoba y La Rioja - ambos sectores; y Santa Fe y Misiones - agua y saneamiento). Similares irregularidades se corroboran en términos de los procedimientos de selección de los directores. En este sentido, en algunas jurisdicciones, se han Ilegado a omitir prácticamente todos los procedimientos previstos en el marco regulatorio (Estado nacional distribución de energía eléctrica) y, en otras, no se ha respetado la composición prevista para este cuerpo en términos de la representación sindical o de usuarios y consumidores (Santa Fe y Misiones, respectivamente - agua y saneamiento).

\footnotetext{
${ }^{21}$ En Argentina, si bien el artículo 42 de la Constitución nacional contempla la participación de las asociaciones de usuarios y consumidores en los organismos de control, existe un debate irresuelto respecto de los alcances de esta participación: comisiones asesoras - consultivas o no $-\mathrm{o}$ directorios.
} 
los entes multisectoriales como en los sectoriales. Las ventajas de los primeros son las menores posibilidades de captura, las economías de escala (burocrática y de desarrollo de capacidades) y la coherencia regulatoria interna; mientras que los entes sectoriales se destacan por su enfoque especializado y por evitar burocracias de gran envergadura (Dourojeanni y Jouravlev, 2003:269). Frente a esta segunda alternativa, es necesario que la normativa establezca interdependencias y sólidas articulaciones entre los organismos responsables de la regulación de sectores vinculados (tales como gas y electricidad). ${ }^{22}$ En este sentido, sería prudente que existiera una instancia colegiada inter-agencias para la resolución de conflictos regulatorios (Abranches, 1999:43). De todo lo anterior, se deduce que no existe una recomendación unívoca sobre la conveniencia de regular por medio de entes sectoriales o multisectoriales. En definitiva, la elección debe ajustarse a las particularidades de cada jurisdicción y, sobre todo, a la estructura y dimensión del sistema de servicios, de forma que, por ejemplo, en los niveles nacionales se aconseja la utilización de entes de regulación sectoriales.

Para concluir, conviene aclarar que las posibilidades de intervención efectiva de los entes reguladores no están restringidas exclusivamente por su diseño institucional. En este sentido, cobra relevancia el entramado institucional en el cual se insertan (relaciones Ejecutivo/Legislativo, independencia del poder judicial etc.) y el orden de prelación en que se materializan las tres principales etapas de todo proceso privatizador: definición del marco regulatorio, creación del ente regulador y transferencia del servicio al nuevo operador. En particular, la secuencia privatizadora emerge como condicionante del buen desempeño de estos organismos (Azpiazu, 2002:88). Por su contribución a la estabilidad jurídica, las prácticas recomendadas son aquellas en las que la creación del marco regulatorio y del ente regulador anteceden al momento de la transferencia de la empresa al sector privado (con independencia de si fue el marco regulatorio o el ente regulador el instituto creado en primera instancia). ${ }^{23}$ De esta forma se evita que las prácticas privadas de prestación creen jurisprudencia contraria a la normativa vigente.

\footnotetext{
${ }^{22}$ Incluso, en el nivel nacional, sería interesante evaluar la viabilidad de crear una "ente regulador de la energía" (Azpiazu, 2002:300).

${ }^{23}$ Resulta clave que el proceso garantice que, en los casos en que el ente preceda al marco, sus funcionarios no puedan luego involucrarse en la definición de la normativa (marco) que afecta su funcionamiento (ente). Asimismo, en términos generales, quienes integren la comisión de privatización no sólo deben poseer calificaciones afines (incluyendo la convocatoria de especialistas) a la tarea que se les encomienda, sino que paralelamente deben estar inhabilitados para conformar los directorios de los entes reguladores o las empresas prestadoras.
} 


\section{7. ¿Quién controla a los organismos reguladores?}

La rendición de cuentas de los entes reguladores es un aspecto clave del proceso regulatorio. Una vez eliminado - tal como aquí se sugirió - el recurso jerárquico ante el Poder Ejecutivo (alzada administrativa), el control de los organismos regulatorios - en función de las disposiciones institucionales de cada país - involucrará la supervisión ejercida en forma permanente por múltiples actores. En todos los casos, el Poder Legislativo - ya sea que actúe ex ante o ex post - y el Poder Judicial - en su carácter de última instancia, deberían velar por el correcto desempeño de estos organismos.

Asimismo, desde mediados de la década del 1950, con los antecedentes de Suecia y Dinamarca, la figura del ombudsman o defensor del pueblo se extendió internacionalmente. Esta institución, que tiene carácter constitucional en muchos países, surge para controlar el ejercicio de las funciones de la administración pública y defender y proteger los derechos y garantías de la población ante hechos, actos u omisiones de la administración. En este sentido, se incorpora como una herramienta más en la que pude descansar el esquema de regulación y control de servicios públicos.

Finalmente, no puede obviarse el papel de la ciudadanía. Sea por medio de comisiones asesoras o mediante la participación en los directorios de los organismos, las asociaciones de usuarios y consumidores se constituyen - en función de sus capacidades de organización, niveles de representatividad, herramientas técnicas y recursos presupuestarios - en observadoras críticas del sistema de prestación, regulación y control $2005 .^{24}$

De esta manera, el funcionamiento de los organismos reguladores descansa sobre un complejo entramado institucional de controles cruzados.

\section{Reflexiones finales}

Como se infiere, el esquema de regulación de los servicios públicos se define en forma asociada a los arreglos institucionales de prestación vigentes en cada momento histórico. Durante el "viejo" período de prestación estatal, la autorregulación se erigió como la forma unívoca de regulación. En el marco de la privatización, la creación de entes reguladores autónomos emergió como el rasgo distintivo del moderno esquema regulatorio. De esta forma, el régimen de

\footnotetext{
${ }^{24}$ Para ampliar, sobre el papel de las asociaciones de defensa del consumidor, consultar Botto, 2005.
} 
regulación se encuentra íntimamente ligado, en cada período histórico, a la forma en que se despliega el Estado en función del régimen de acumulación vigente.

Puesto que las prioridades del proceso de enajenación de activos no estuvieron vinculadas primariamente a motivaciones sectoriales, el aspecto regulatorio del programa privatizador fue uno de los elementos más endebles del cambio de roles público/privado: el diseño de los entes ha sido descuidado, de forma que el alcance de sus atribuciones se encontró limitado por ambigüedades normativas y deficiencias estructurales. En este sentido, la debilidad de la regulación pública se convirtió en un elemento funcional a los intereses de los consorcios operadores. Así, tal como los procesos de privatización se han alejado de las recomendaciones de la literatura en la materia, el diseño y la práctica regulatoria también han diferido enormemente de tales prescripciones.

Revertir esta pesada herencia requiere revalorizar los alcances de los entes, priorizando su capacidad técnica y autonomía. Sin avasallar las funciones del Poder Ejecutivo, responsable de las políticas de planificación, los entes - tanto frente a la prestación pública como privada - deberían, de una vez por todas, comenzar a definir los aspectos regulatorios clave de los servicios públicos de infraestructura. En definitiva, se trata - precisamente - de que los entes reguladores tengan capacidades reales de regulación.

\section{Referencias bibliográficas}

ABAR (Associação Brasileira de Agências de Regulação). Agencias reguladoras. Instrumentos de fortalecimiento del Estado. Porto Alegre: Abar, 2003.

ABRANCHES, S. Reforma regulatória: conceitos, experiências e recomendações. Revista do Serviço Público, v. 50, n. 2. p. 19-49, abr./jun. 1999.

ACIJ (Asociación Civil por la Igualdad y la Justicia). Conflicto en puerta por AySA. Disponible en: <www.acij.org.ar/mostrar_noticia.php?id=356>.

AZPIAZU, D. Las privatizaciones en Argentina. ¿Precariedad regulatoria o regulación funcional a los privilegios empresarios? Revista Ciclos, Buenos Aires, v. 11, n. 21, primer semestre 2001.

. Las privatizaciones en Argentina. Diagnósticos y propuestas para una mayor competitividad y equidad social. Buenos Aires: Osde, Ciepp, 2002.

; SCHORR, M. Desempeño reciente y estructura del mercado gasífero argentino: asimetrías tarifarias, ganancias extraordinarias y concentración del capital. Buenos Aires: Flacso, 2001. (Documento de Trabajo n. 1). 
BASUALDO, E. Estudio de historia económica argentina. Desde mediados del siglo XX a la actualidad. Buenos Aires: Siglo Veintiuno Editores, 2006.

BOTTO, A. P. Las organizaciones y la lucha por los derechos de los consumidores: ¿mito o realidad? La regulación de los servicios públicos en Argentina y Brasil. 2005. Tesis (Maestría en Sociología Económica) — Instituto de Altos Estudios Sociales, Universidad Nacional de San Martín, Buenos Aires.

CEPAL (Comisión Económica para América Latina y el Caribe). La privatización de servicios públicos basados en agua. LC/R. 1486. 1995.

DOUROJEANNI, A.; JOURAVLEV, A. Regulación de servicios de agua potable. In: Agua para las Américas en el Siglo XXI. México DF: El Colegio de México, Comisión Nacional de Agua, 2003.

FIEL (Fundación de Investigaciones Económicas Latinoamericanas). La regulación de la competencia y de los servicios públicos. Teoría y experiencia argentina reciente. Buenos Aires: Fiel, 1999.

GÓMEZ, P. Eficiencia regulatoria. In: FORO REGULATORIO. Anales... Caracas: Iesa, 31 oct. 2006.

GROISMAN, E. Servicio público. Un concepto polémico. Revista Encrucijadas, Buenos Aires, v. 1, n. 6, 2001.

HERRERO, F. La regulación provincial de los servicios públicos en Argentina. La Plata: Ediciones de la UNLa, 2002.

ILPES (Instituo Latinoamericano y del Caribe de Planificación Económica y Social). Diseño estratégico e infraestructura básica. Análisis comparativo. In: Diseño estratégico e infraestructura básica. Santiago de Chile: Ilpes, 1999.

LOPEZ, A.; FELDER, R. Regulación y control de la calidad de los servicios públicos privatizados: los casos del servicio de gas natural, electricidad y agua potable. Buenos Aires: Inap, 1996. (Documento no 57.)

MAJONE, G.; LA SPINA, A. El Estado regulador. Gestión y Política Pública, v. 2, n. 2, jul./ dic. 1993.

MELO, J. R. El Estado y el desarrollo de la infraestructura. In: Diseño estratégico e infraestructura básica. Santiago de Chile: Ilpes. 1999.

. Política regulatoria: uma revisão da literatura. Revista Brasileira de Informação Bibliográfica em Ciencias Sociais, n. 50. p. 7-44, 2000.

. As agências regulatórias: gênese, desenho institucional e governança. In: ABRUCIO, F; LOUREIRO, M. R. (Orgs.). O Estado numa era de reformas: os anos FHC. Brasília: SegesMP, 2002. p. 247-305. 
NAHÓN, C. La metamorfosis del Estado. Límites y potencialidades de la regulación pública. In: PRIMERAS JORNADAS DE ESTUDIOS SOCIALES DE LA ECONOMÍA. Anales... Buenos Aires: Centro de Estudios Sociales de la Economía, Instituto de Altos Estudios Sociales, Universidad Nacional de San Martín. 19 y 20 jul. 2006.

. Privatizaciones en América Latina y el Caribe: un estado de la cuestión. Revista Nueva Sociedad, Buenos Aires, n. 207, ene./feb. 2007. Disponible en: <www.nuso.org/ upload/anexos/nahon.pdf>.

ORLANSKY, D. Las políticas de descentralización. Revista Desarrollo Económico, Buenos Aires, v. 38, n. 151, p. 827-844, oct./dic. 1998.

PHILLIPS, C. F. JR. The regulation of public utilities. Theory and practice. Arlington: Public Utilities Report, Inc. 1993.

PORTO, A.; URBIZTONDO, S. Regulación económica: un enfoque "principal-agente" de la relación entre el Estado, el regulador y la empresa regulada. Estudios de Economía, v. 20, n. 2, 1993.

ROSS, S. The economic theory of agency: the principal's problem. American Economic Review, v. 63, 1973.

SPILLER, P. El por qué de la regulación de los servicios públicos. FGYS, dic. 1998. (Cuaderno no 2.)

STARK, C. Regulación, agencias reguladoras e innovación de la gestión pública en América Latina. In: SPINK, P. et al. Nueva gestión pública y regulación en América Latina. Balances y desafíos. Caracas: Clad, 2001.

URBIZTONDO, S.; ARTANA, D.; NAVAJAS, F. La autonomía de los nuevos entes reguladores argentinos. Revista Desarrollo Económico, Buenos Aires, v. 38, p. 7-40, otoño 1998.

UUC (Unión de Usuarios y Consumidores). Usuarios en retroceso. Nuevo marco regulatorio para AySA. UUC, 2006. Disponible en: <www.usuarios.org.ar/>.

VICKERS, J.; YARROW, G. Un análisis económico de la privatización. México: Fondo de Cultura Económica, 1991.

VISPO, A. Los entes de regulación. Problemas de diseño y contexto. Aportes a un necesario debate en al Argentina de fin de siglo. Buenos Aires: Grupo Editorial Norma/Flacso, 1999. 